Journal of Algebraic Combinatorics, 20, 5-16, 2004

\title{
On Certain Coxeter Lattices Without Perfect Sections
}

ANNE-MARIE BERGÉ

berge@math.u-bordeaux.fr

Laboratoire A2X, Mathématiques, Université Bordeaux 1, 351, cours de la Libération,

33405 TALENCE Cedex, France

Received December 6, 2002; Revised May 12, 2003; Accepted June 5, 2003

\begin{abstract}
In this paper, we compute the kissing numbers of the sections of the Coxeter lattices $\mathbb{A}_{n}^{\frac{n+1}{2}}, n$ odd, and in particular we prove that for $n \geq 7$ they cannot be perfect. The proof is merely combinatorial and relies on the structure of graphs canonically attached to the sections.
\end{abstract}

Keywords: perfect lattice, kissing number, bipartite graph

\section{Introduction}

A problem of recent interest is to construct integral perfect lattices with odd norm. By lattice we mean an additive subgroup $L$ of a Euclidean space $(E, \cdot)$ which is additively generated by some $\mathbb{R}$-basis for $E$. Such a lattice is integral if the inner product $x \cdot y$ takes integral values on it. The norm of a lattice $L$ is the minimal value $M$ of $x \cdot x$ for $x \in L, x \neq 0$, and the vectors $\pm x \in L$ for which $x \cdot x=M$ are the minimal vectors of $L$. Their number $2 s$ is the kissing number of $L$, terminology which refers to the sphere packing classically associated to the lattice $L$.

Perfect lattices arise in determining the densest lattice packing of spheres. A lattice $L$ is perfect if it is uniquely determined up to similarity by the coordinates of its minimal vectors in one of its $\mathbb{Z}$-bases. In 1877 Korkine and Zolotareff proved that all lattices whose packing density is a local maximum (extreme lattices) are perfect. They also proved that a perfect lattice can be rescaled so as to be integral, and that its kissing number $2 s$ satisfies

$$
s \geq \frac{n(n+1)}{2}
$$

where $n=\operatorname{dim} E$. All similarity classes of perfect lattices are now known up to dimension 7. From dimension 8 onwards, the complete classification seems out of reach. Voronoi's algorithm for perfect forms produced at this date 10916 inequivalent forms of dimension eight (for a catalogue, see http://www.math.u-bordeaux.fr $/ \sim$ martinet/).

An intriguing property of this list is that it contains no integral lattice of odd norm. It has recently been proved by Martinet and Venkov that the lattice $P_{7}^{2}$ (in the notation of 
[4]) is the unique integral perfect lattice of dimension $2 \leq n \leq 9$ having norm 3 ([7]). Their method consists in finding for the kissing number of integral lattices of norm 3 an upper bound strictly inferior to $n(n+1)$. Note that a first 10-dimensional example of a perfect lattice having odd norm (namely 11) was recently constructed by Martinet (see [3], Section 4).

A natural method to construct integral perfect lattices having odd norm would consist in taking sections of a known one that contain a great number of its minimal vectors. About this method by sections, note that the algorithms of Batut and Martinet to "X-ray" integral lattices ([1]) showed that out of the known perfect lattices of dimension $3 \leq n \leq 8, P_{7}^{2}$ is also the unique one without perfect sections of dimension $>1$.

This remarkable lattice $P_{7}^{2}$ belongs to an infinite sequence of perfect lattices with odd norm (when rescaled to be integral). This sequence is part of a family that Coxeter derived from the root lattices $\mathbb{A}_{n}$ (see [6], Section 5.2): for any dimension $n \geq 1$ and any divisor $q$ of $n+1$ the lattice $\mathbb{A}_{n}^{q}$ is the unique sublattice of the dual lattice $\mathbb{A}_{n}^{*}$ that contains $\mathbb{A}_{n}$ to index $q$. For $n>5$ and $q<\frac{n+1}{2}$, all these lattices have the same norm as $\mathbb{A}_{n}$, and are therefore perfect (and even extreme) with even norm when rescaled so as to be integral. For $q=\frac{n+1}{2}$ ( $n$ odd, $n \geq 5$ ), the Coxeter lattices are extreme too but with norm $\frac{2 n-2}{n+1}<2$, and their primitive integral copy has odd norm if and only if $n \equiv 3 \bmod 4$. The aim of this paper is to X-ray these lattices. In particular, as a direct consequence of the combinatorial Theorem 2 (stated and proved in Section 4), we find that for $n \geq 7$, any section of $L=\mathbb{A}_{n}^{\frac{n+1}{2}}$ of dimension $r, 1<r<n$, contains at most $r(r-1)+2<r(r+1)$ minimal vectors of $L$. This enables us to extend to every odd dimension the property of "emptiness" noticed for the lattice $P_{7}^{2} \sim \mathbb{A}_{7}^{4}$ :

Theorem 1 In everyodd dimension $n \geq 3$, the Coxeter lattice $\mathbb{A}_{n}^{\frac{n+1}{2}}$ has no perfect section of the same norm in dimension $>1$, except the lattice $\mathbb{A}_{5}^{3}$ which possesses 15 planar hexagonal sections.

In Section 2 we give a description of the lattice $\mathbb{A}_{n}^{\frac{n+1}{2}}$ which leads to a combinatorial approach of the determination of its sections with best kissing number; this combinatorial problem is interpreted in Section 3 in terms of graphs, and solved in Section 4.

I want to thank J. Martinet for the motivation of this work, and the Reviewers for helpful suggestions and corrections.

\section{A conjecture of Martinet}

Let $E$ be a Euclidean space of dimension $n$, and let $\left(e_{1}, \ldots, e_{n}\right)$ be a basis for the dual lattice $\mathbb{A}_{n}^{*}$ with Gram Matrix

$$
\frac{1}{n+1}\left(\begin{array}{ccccc}
n & -1 & -1 & \ldots & -1 \\
-1 & n & -1 & \ldots & -1 \\
\cdot & \cdot & \cdot & \ldots & \cdot \\
-1 & -1 & -1 & \ldots & n
\end{array}\right)
$$


the minimal vectors of $\mathbb{A}_{n}^{*}$ are the $\pm e_{i}, 0 \leq i \leq n$, where $e_{0}=-\left(e_{1}+e_{2}+\cdots+e_{n}\right)$. One possible definition of the Coxeter lattice is

$$
\mathbb{A}_{n}^{\frac{n+1}{2}}=\left\{x_{1} e_{1}+x_{2} e_{2}+\cdots+x_{n} e_{n} \mid\left(x_{i}\right) \in \mathbb{Z}^{n} \text { and } \sum_{i} x_{i} \equiv 0 \bmod 2\right\},
$$

as the right-hand side defines a sublattice of index 2 in $\mathbb{A}_{n}^{*}$ containing the root lattice $\mathbb{A}_{n}=\left\langle e_{i}-e_{0}, 1 \leq i \leq n\right\rangle$. It then has norm $\frac{2 n-2}{n+1}$ and its minimal vectors are $\pm\left(e_{i}+e_{j}\right)$, $0 \leq i<j \leq n$. So, to establish Theorem 1 we shall bound the number of these vectors contained in a given strict subspace of $E$, discarding its Euclidean structure.

In the following, $E_{n}$ is a real vector space of dimension $n \geq 2$ equipped with a basis $\left(e_{1}, e_{2}, \ldots, e_{n}\right)$. Put

$$
e_{0}=-\left(e_{1}+e_{2}+\cdots+e_{n}\right)
$$

For a subspace $F$ of $E_{n}$ we consider its subset

$$
S_{F}=F \cap\left\{e_{i}+e_{j}, 0 \leq i<j \leq n\right\},
$$

with cardinality

$$
s_{F}=\left|S_{F}\right| \text {. }
$$

Example A subspace $F$ of $E_{n}$ is said canonical if it is spanned by some vectors $e_{i}, 0 \leq$ $i \leq n$.

For a canonical subspace $F \subset E_{n}$ of rank $r(1 \leq r \leq n-1)$ we have $s_{F}=\frac{r(r-1)}{2}$ if $r \neq n-1$ and $s_{F}=\frac{r(r-1)}{2}+1$ if $r=n-1$. Indeed, up to permutations by the symmetric group $S_{n+1}$ we may assume $F=\left\langle e_{0}, e_{1}, \ldots, e_{r-1}\right\rangle$. It then contains the $\left(\begin{array}{c}r \\ 2\end{array}\right)$ vectors $e_{i}+e_{j}$, $0 \leq i<j \leq r-1$, and no more except if $r=n-1$, when we must add the vector $e_{n-1}+e_{n}=-e_{0}-e_{1}+\cdots-e_{n-2}$.

For any dimension $n \geq 3$ and any integer $r, 1 \leq r \leq n-1$, we define

$$
s_{n}(r)=\max _{F \subset E_{n}, \operatorname{dim} F=r} s_{F} .
$$

Martinet ([5]) stated the following:

\section{Conjecture}

1. For $r \geq 5, s_{n}(r)$ is equal to either $\frac{r(r-1)}{2}$ or $\frac{r(r-1)}{2}+1$ according as $r \neq n-1$ or $r=n-1$.

2. For $n \geq 5$ and $r \geq 2$, we have $s_{n}(r)<\frac{r(r+1)}{2}$ except for $(n, r)=(5,2)$, where $s_{5}(2)=3$. 
The second part of this conjecture, applied to our lattice problem, implies Theorem 1 , the value $s_{5}(2)$ corresponding to the hexagonal sections of the lattice $\mathbb{A}_{5}^{3}$, which are perfect indeed.

The conjecture will result of the actual determination of all values of $s_{n}(r)$ and of the subspaces $F$ which realize them. To state and prove these results, an interpretation in terms of graphs is needed.

\section{Graphs associated with a subspace $F$ of $E_{n}$}

The bounds of $s_{F}$ are attained at subspaces $F$ of $E$ generated by their subsets $S_{F}$; from now on we only consider such subspaces.

Definition 1 With a subspace $F$ of $E$ we associate the graph $G=G_{F}$ of the relation $e_{i}+e_{j} \in F$ : its vertex set is $\{0,1, \cdots, n\}$, and two vertices $i$ and $j$ are joined if $e_{i}+e_{j}$ lies in $F$.

To any basis $\mathcal{B} \subset S_{F}$ of $F$ we attach the subgraph $G_{\mathcal{B}} \subset G_{F}$ obtained by keeping only the edges $i j$ of $G_{F}$ such that $e_{i}+e_{j} \in \mathcal{B}$.

Our aim is to compare the number of edges $s_{F}$ of $G_{F}$ with the number of edges $r=\operatorname{dim} F$ of $G_{\mathcal{B}}$.

Example For a canonical subspace $F$ of dimension $r, 3 \leq r \leq n-1$, there is a basis $\mathcal{B}$ whose graph is a triangle linked to a path: for instance the vectors $e_{0}+e_{1}, e_{1}+e_{2}, e_{2}+$ $e_{0}, e_{2}+e_{3}, \ldots, e_{r-2}+e_{r-1}$ constitute a basis for $F=\left\langle e_{0}, \ldots, e_{r-1}\right\rangle$.

We now discuss the existence of cycles in the graphs $G_{F}$ and $G_{\mathcal{B}}$.

\section{Lemma 1}

1. If the vertices $i$ and $j$ are connected in $G_{F}$ by a path of odd length, ij is an edge of $G_{F}$.

2. The graph $G_{\mathcal{B}}$ does not contain an even cycle of length $\geq 4$.

3. If a connected component $C$ of $G_{F}$ contains an odd cycle, then all the vectors $e_{i}, i \in C$ belong to $F$, and $C$ is a complete graph.
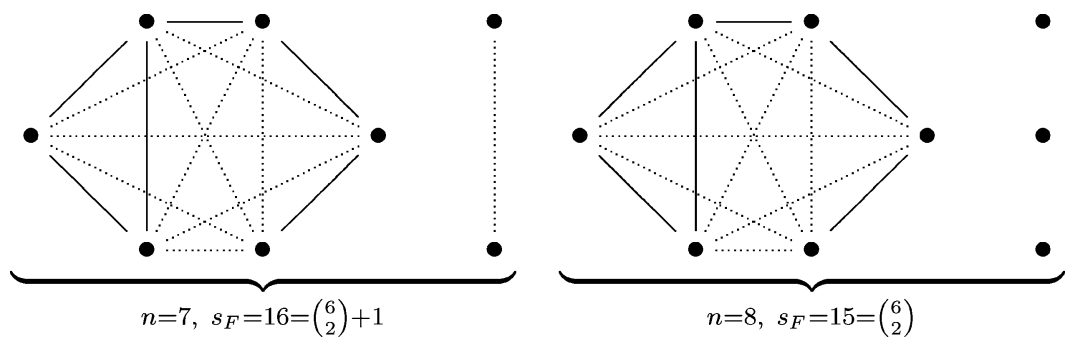

Figure 1. Graphs $G_{\mathcal{B}}$ and $G_{\mathcal{F}}$ for canonical subspaces of dimension 6 (the $s_{F}-r$ edges of $G_{F} \backslash G_{\mathcal{B}}$ appear in dotted lines). 
Proof: By induction from the following relations, where $i, j, k, l \in\{0,1, \ldots, n\}$ :

$$
\begin{aligned}
\left(e_{i}+e_{j}\right) & =\left(e_{i}+e_{l}\right)+\left(e_{j}+e_{k}\right)-\left(e_{k}+e_{l}\right), \\
e_{i} & =\frac{1}{2}\left(\left(e_{i}+e_{j}\right)-\left(e_{j}+e_{k}\right)+\left(e_{i}+e_{k}\right)\right), \\
e_{i} & =\left(e_{i}+e_{j}\right)-e_{j} .
\end{aligned}
$$

We can now characterize the canonical subspaces by their graphs.

Lemma 2 Let $F$ be an $r$-dimensional subspace of $E_{n}(3 \leq r \leq n-1)$. Then $F$ is canonical if and only if its graph $G_{F}$ contains a complete $r$-graph, i.e. a graph with $r$ vertices and $\left(\begin{array}{c}r \\ 2\end{array}\right)$ edges.

Proof: We have already seen that if $F$ is canonical, its whole graph consists of a complete $r$-graph and a path of length 1 (resp. $n+1-r$ isolated vertices) if $r=n-1$ (resp. $r<n-1$ ).

Conversely, suppose that there is in $G_{F}$ a connected component $C$ with $|C|=r$ vertices and $\left(\begin{array}{l}r \\ 2\end{array}\right)$ edges. Since $C$ is complete of order $r \geq 3$, it contains at least one triangle; it follows from the third part of Lemma 1 that all $e_{i}, i \in C$ belong to $F$. Since $|C|=\operatorname{dim} F$, we conclude that $F=\left\langle e_{i}, i \in C\right\rangle$.

\section{Calculation of $s_{n}(r)$.}

Linear type. Let $F$ be a strict subspace of $E_{n}$ and let $G_{F}=\bigcup_{C \in \mathcal{C}} C$ the partition of its graph into connected components. We say that the component $C \in \mathcal{C}$ is of linear type if the subspace

$$
F_{C}=\left\langle e_{i}+e_{j} \text { with } i j \text { edge of } C\right\rangle
$$

of $F$ admits a basis $\mathcal{B}_{C}$ whose graph is a path.

We say that $F$ itself is of linear type if, apart from isolated vertices, every component of $G_{F}$ is of linear type. We label the type by the sequence of the lengths of the paths, the zeros representing the isolated vertices.

For example, figure 2 shows the four possible graph structures for $r=2$ (the graph of a basis $\mathcal{B} \subset \cup \mathcal{B}_{C}$ appears in continuous lines).

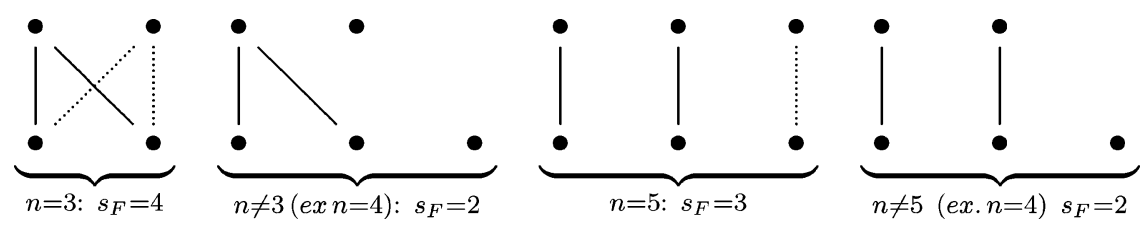

Figure 2. Linear types [2], [2, 0, 0], [1, 1, 1] and [1, 1, 0]. 
Theorem 2 below shows in particular that the invariant $s_{F}$ assumes its greatest value for subspaces which are either canonical or (in low dimension) of linear type.

Theorem 2 Let $F$ be an $r$-dimensional $(1 \leq r \leq n-1)$ subspace of $E_{n}$. Then

1. For $r \geq 4$, we have

$$
s_{F} \leq \begin{cases}\frac{r(r-1)}{2} & \text { if } r \neq n-1, \\ \frac{r(r-1)}{2}+1 & \text { if } r=n-1,\end{cases}
$$

except for $r=4, n=5, F$ of linear type [4] where $s_{F}=9$. Equality in (1) holds only when $F$ is either canonical or of one of the following linear types:

$r=4: n \geq 6$, type $[4,0,0, \ldots]$ or $n=7$, type $[3,1,1]$

$r=5: n=7$, type $[5,1]$

$r=6: n=7$, type [6].

2. For $r=1$,

$n \neq 3: s_{n}(1)=1$ attained at type $[1,0, \ldots]$,

$n=3: s_{3}(1)=2$ attained at type $[1,1]$.

3. For $r=2$,

$n \neq 3,5: s_{n}(2)=2$, at types $[1,1,0, \ldots]$ and $[2,0, \ldots]$,

$s_{3}(2)=4$ attained at type [2],

$s_{5}(2)=3$ attained at type $[1,1,1]$.

4. For $r=3$,

$n \neq 5: s_{n}(3)=4$, attained at linear types $[3,0,0, \ldots]$ and $[1,1,1,1]($ if $n=7)$, and at canonical hyperplanes (if $n=4$ );

$n=5: s_{5}(3)=5$ attained at type $[3,1]$.

Going back to Euclidean lattices we can interpret some maximal values of $s$ in low dimensions. We first note that the value $s_{3}(2)$ corresponds to square sections of the cubic lattice $\mathbb{A}_{3}^{2}$, the set $S_{F}$ consisting of two pairs of orthogonal vectors. The sections of $\mathbb{A}_{5}^{3}$ which realize the maximum $s_{5}(2)=3$ (resp. $s_{5}(4)=9$, resp. $s_{5}(3)=5$ ) are similar to the perfect lattice $\mathbb{A}_{2}$ (resp. to $\mathbb{A}_{2} \otimes \mathbb{A}_{2}$, resp. to the "fragile" lattice of crystallography, see [6], Section 9.5). In dimension 7 , there are coincidences, due to the multiple embeddings of the lattice $\mathbb{A}_{7}^{4} \sim \mathbb{E}_{7}^{*}$ into $\mathbb{A}_{7}^{*}$; for instance, canonical as well as linear type [6] hyperplanes correspond to sections of $\mathbb{E}_{7}^{*}$ similar to the isodual lattice $\mathbb{D}_{6}^{+}$. This phenomenon does not occur for $n=9$.

The rest of the paper is devoted to the proof of Theorem 2. Let

$$
G_{F}=\bigcup_{C \in \mathcal{C}} C
$$

be the partition of the graph of $F$ into connected components, where at most one $C$ is complete with $|C| \geq 3$ (by Lemma 2 it corresponds to the canonical subspace $F_{C}=\left\langle e_{i}, i \in\right.$ $C\rangle$ of $F$ ). 

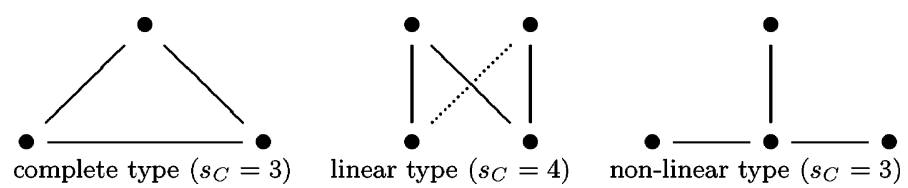

Figure 3. Connected components $C$ such that $r_{C}=3$.

For a component $C \in \mathcal{C}$ we denote by

$c=|C|$ the number of vertices of $C$,

$s_{C}$ the number of edges of $C$ (or size of $C$ ),

$r_{C}$ (rank of $C$ ) the dimension of $F_{C}=\left\langle e_{i}+e_{j}, i j\right.$ edge of $\left.C\right\rangle$. (Of course for isolated vertices $c=1$ and $s_{C}=r_{C}=0$.)

For example there are three possible components of rank 3 .

Contribution of a component. Lemma 2 settles this question for complete components. We now describe the other cases.

Lemma 3 Let $C$ be a non-complete component of $G_{F}$, with $c \geq 2$.

1. There exists an integer $d_{C}, 0 \leq d_{C} \leq c-2, d_{c} \equiv c \bmod 2$, such that

$$
s_{C}=\frac{c^{2}-d_{C}^{2}}{4} \leq\left\lfloor\frac{c^{2}}{4}\right\rfloor
$$

2. $F_{C}$ admits a basis whose graph is a path linked to a star of degree $d_{C}+1$, and its dimension is

$$
r_{C}= \begin{cases}c-1 & \text { if } c \leq n \\ c-2 & \text { if } c=n+1\left(\text { which requires } n \text { odd and } d_{C}=0\right)\end{cases}
$$

3. $s_{C}=\left\lfloor\frac{c^{2}}{4}\right\rfloor$ only if $C$ is of linear type.

4. The following conditions are equivalent:

(i) $\sum_{i \in C} e_{i} \in F_{C}$

(ii) $d_{C}=0$

(iii) $C$ is of linear type with an even number of vertices.

\section{Proof:}

1. Since $C$ is not complete, it does not contain odd cycles. It is thus bipartite (see [2], I.2, Theorem 4), and even by Lemma $1, C$ is a complete bipartite graph, i.e. there exists a partition $C=V_{0} \cup V_{1}$ of $C$ such that $i j$ is an edge of $C$ if and only if $i$ and $j$ are in distinct sets $V_{k}$, as we now prove. Indeed, given $i, j \in C$, the lengths of two paths $i-j$ are congruent modulo 2 (otherwise, they would form an odd cycle); then $V_{0}$ and 
$V_{1}$ are the equivalence classes for the equivalence relation $i \mathcal{R} j$ if $i=j$ or if $i$ and $j$ are connected by an even path. Clearly two neighbours in $C$ belong to distinct classes; conversely, if $i$ and $j$ are in distinct classes, there are connected by a path of odd length, and by Lemma $1, i j$ is an edge of $C$. We conclude that $s_{C}=\left|V_{0}\right|\left|V_{1}\right|=\frac{c+d_{C}}{2} \frac{c-d_{C}}{2}$ where $d_{C}=|| V_{0}|-| V_{1}||$; thus we recover Mantel's bound $\left\lfloor c^{2} / 4\right\rfloor$ for graphs without triangles.

2. From Lemma 1 it follows that the subgraph $G_{\mathcal{B}}$ associated with any basis of $F_{C}$ does not contain any cycle. Thus its connected components are trees, $G_{\mathcal{B}}=T_{1} \cup T_{2} \cup \cdots \cup T_{m}$ say. We then have $r_{C}=\sum_{i}\left(\left|T_{i}\right|-1\right)=\left|G_{\mathcal{B}}\right|-m \leq c-1$. Actually, in the case $c=n+1$ (i.e. $G_{F}=C$ ), we must have $r<n=c-1$, since otherwise $F_{C}=E_{n}$ would be canonical. We now define for $F_{C}$ a standard basis $\mathcal{B}_{C}$ whose graph is a tree depending only on $d_{C}$.

Put $c=2 p+d_{C}$ so that the vertex classes of $C$ have respectively $p$ and $p+d_{C}$ elements; up to permutation by $S_{n+1}$ we may assume them to be

$$
\{2 k-1,1 \leq k \leq p\} \quad \text { and } \quad\{2 k-2,1 \leq k \leq p\} \cup\left\{2 p+k, 0 \leq k \leq d_{C}-1\right\} .
$$

Then the subspace $F_{C}$ contains the following $c-1$ vectors:

$$
f_{i}=\left\{\begin{array}{cc}
e_{i-1}+e_{i} & \text { for } 1 \leq i \leq 2 p-1 \\
e_{2 p-1}+e_{i} & \text { for } 2 p \leq i \leq c-1
\end{array}\right.
$$

For any $\left(\lambda_{i}\right) \in \mathbb{R}^{c-1}$ we have

$$
\sum_{i=1}^{c-1} \lambda_{i} f_{i}=\lambda_{1} e_{0}+\sum_{1}^{2 p-2}\left(\lambda_{i}+\lambda_{i+1}\right) e_{i}+\left(\sum_{2 p-1}^{c-1} \lambda_{i}\right) e_{2 p-1}+\sum_{2 p}^{c-1} \lambda_{i} e_{i} .
$$

For any $\lambda \in \mathbb{R}$ we then have the equivalence

$$
\sum_{i=1}^{c-1} \lambda_{i} f_{i}=\lambda \sum_{i \in C} e_{i} \Leftrightarrow \begin{cases}\lambda_{i}=0 & \text { if } i \in\{1, \ldots, 2 p-1\} \text { is even, } \\ \lambda_{i}=\lambda & \text { if } i \in\{1, \ldots, 2 p-1\} \text { is odd } \\ & \text { or if } i \geq 2 p, \\ d_{C} \lambda=0 . & \end{cases}
$$

If $c \leq n$, the $e_{i}, i \in C$, are independent, thus from $\left(^{*}\right)$ with $\lambda=0$ we obtain that the $c-1$ vectors $f_{i}$ are independent, and since $r_{C} \leq c-1$, they constitute a basis for $F_{C}$, whose rank is $r_{C}=c-1$.

If $c=n+1$, we know that $r_{C} \leq c-2$, and the $c-1$ vectors $f_{i}$ must satisfy a nontrivial relation $\sum_{1 \leq i \leq c-1} \lambda_{i} f_{i}=0$. On the other hand, there exists, up to multiplication by a scalar, a unique non-trivial relation between the $e_{i}, i \in C: e_{0}+e_{1}+\cdots+e_{n}=0$. Therefore, using $\left(^{*}\right)$ with $\lambda \neq 0$, we obtain $d_{C}=0$ and thus $n=2 p-1$. Conversely, if $d_{C}=0$, the $n$ vectors $f_{i}=e_{i-1}+e_{i}, i=1, \ldots, n$ satisfy the "unique" relation $f_{n}=-f_{1}-f_{3}-\cdots-f_{n-2}$, and $f_{1}, f_{2}, \ldots, f_{n-1}$ constitute a basis for $F_{C}=F$. Its graph is a path of $c-1=n$ vertices (which does not span $C$ ). 
3. It is clear from the previous parts of the lemma, as $s_{C}$ attains Mantel's bound if and only if $d_{C}=0$ or 1 .

4. It follows immediately from $\left(^{*}\right)$ with $\lambda=1$.

We now compare Mantel's bound $\left\lfloor\frac{c^{2}}{4}\right\rfloor$ to $\left(\begin{array}{c}r_{C} \\ 2\end{array}\right)$. The differences $\left(\begin{array}{c}r_{C} \\ 2\end{array}\right)-\left\lfloor\frac{\left(r_{C}+1\right)^{2}}{4}\right\rfloor$ and $\left(\begin{array}{c}r_{C} \\ 2\end{array}\right)-\frac{\left(r_{C}+2\right)^{2}}{4}$ are increasing functions of $r_{C}$. We can thus state the following.

Lemma 4 Let $C$ be a non-complete component of $G_{F}$ of positive rank. Its size $s_{C}$ and rank $r_{C}$ satisfy

$$
s_{C}-\left(\begin{array}{c}
r_{C} \\
2
\end{array}\right)=\left\{\begin{array}{cl}
3 & \text { if }\left(n, r_{C}\right)=(3,2) \text { or }(5,4), \\
1 & \text { if }\left(n, r_{C}\right)=(7,6) \text { or if } r_{C} \leq 3 \text { (linear type) } \\
0 \quad & \text { if } C \text { is complete, or if } r_{C}=3 \text { (non-linear type) } \\
& \text { or if } \left.r_{C}=4 \text { (linear type, } n \geq 6\right),
\end{array}\right.
$$

and $s_{C}<\left(\begin{array}{c}r_{C} \\ 2\end{array}\right)$ otherwise.

Right now, Theorem 2 is proved for subspaces $F$ whose graphs contain exactly one component of positive rank. In particular these $F$ realize the bounds $s_{3}(2)$ (figure 2), $s_{n}(4)$ (figure 4) and $s_{7}(6)$ (figure 5).

From now on, we suppose that the graph $G_{F}=\bigcup_{C \in \mathcal{C}} C$ of $F$ contains at least two components of positive rank.

Dimensions. Consider $x=e_{i}+e_{j} \in S_{F}$. The indices $i$ and $j$ belong to the same connected component $C$, and thus the vector $x$ belongs to the corresponding subspace $F_{C}$. Since $S_{F}$ spans $F$, we have $F=\sum F_{C}$. We first discuss whether this sum is direct.

Lemma 5 We have $r=\sum_{C} r_{C}-\delta$ with $\delta=0$ or 1 , where $\delta=1$ if and only if all non-complete components $C \in \mathcal{C}$ have linear type and odd rank.

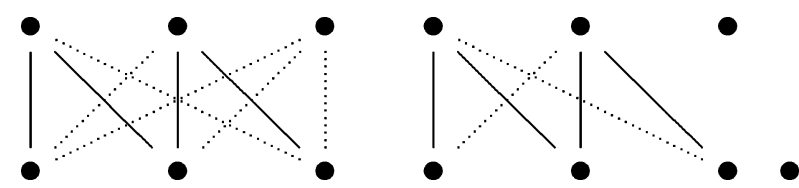

Figure 4. Linear types $[4]\left(s_{F}=9\right)$ and $[4,0,0]\left(s_{F}=6\right)$.

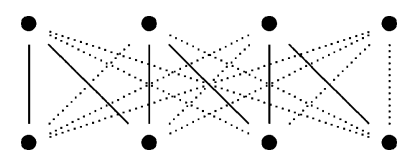

Figure 5. Linear type [6] $\left(s_{F}=16\right)$. 
Proof: Note that for $|C| \geq 2$ the typical vector of $F_{C}$ can be written $x_{C}=\sum_{i \in C} \lambda_{i} e_{i}$. Since any relation of dependence between the $e_{i}$ has the form $\lambda\left(e_{0}+e_{1}+\cdots+e_{n}\right)=0$ for some $\lambda \in \mathbb{R}$, and since $\mathcal{C}$ is a partition of $\{0,1, \ldots, n\}$, we have the following equivalence:

$$
\left(\sum_{C \in \mathcal{C}} x_{C}=0, x_{C} \in F_{C}\right) \Longleftrightarrow\left(\exists \lambda \in \mathbb{R} \mid \forall C \in \mathcal{C}: x_{C}=\lambda \sum_{i \in C} e_{i}\right) .
$$

Using the last part of Lemma 3, we are left with two possibilities:

(1) there is an isolated vertex or a non-complete component with invariant $d_{C} \neq 0$ : the above $\lambda$ is null, and $F=\oplus F_{C}$.

(2) every non-complete component $C$ has order $|C| \geq 2$ and invariant $d_{C}=0$ : then for all $C \in \mathcal{C}$, the nonzero vector $x_{C}=\sum_{i \in C} e_{i}$ belongs to $F_{C}$, and we have a nontrivial relation $\sum x_{C}=0$. As this is up to scale the only one, we have $\operatorname{dim}\left(\sum F_{C}\right)=$ $\sum \operatorname{dim} F_{C}-1$.

Proof of Theorem 2: We have to compare the size $s_{F}=\sum r_{C}$ of $G_{F}$ to the dimension $r=\sum r_{C}-\delta$ of $F$.

Type $[1, \ldots, 1,0, \ldots, 0]$ case: the graph $G_{F}$ consists of $1 \leq k \leq \frac{n+1}{2}$ paths of length 1 and of $n+1-k$ isolated vertices; it then has $s_{F}=k$ edges, while by Lemma $5, r=k-\delta$, where $\delta=1$ if and only if there are no isolated vertices i.e. $n=2 k-1=2 r+1$. Thus

$$
s_{F}=r+\delta= \begin{cases}r & \text { if } n \geq 2 r, n \neq 2 r+1 \\ r+1 & \text { if } n=2 r+1\end{cases}
$$

is $<\left(\begin{array}{c}r \\ 2\end{array}\right)$ if and only if $r \geq 4$. In contrast, this linear type realizes the values $s_{n}(1), s_{n}(2)$ (in particular $s_{5}(2)=3$ ) and $s_{7}(3)$.

General case: $\max _{C} r_{C}=r_{0} \geq 2$. From Lemma 4 we deduce

$$
s_{F}=\sum s_{C} \leq \sum\left(\begin{array}{c}
r_{C} \\
2
\end{array}\right)+k
$$

where $k$ denotes the number of components $C$ of linear type and rank $r_{C} \leq 3$. Now, writing $\sum\left(r_{C}^{2}-r_{C}\right)=\left(\sum r_{C}\right)^{2}-\sum r_{C}-2 \sum_{C \neq C^{\prime}} r_{C} r_{C^{\prime}}$ where $\sum r_{C}=r+\delta$ by Lemma 5 , we obtain the inequality

$$
\left(\begin{array}{l}
r \\
2
\end{array}\right)-s_{F} \geq \sum_{C \neq C^{\prime}} r_{C} r_{C^{\prime}}-k-\delta r
$$

which, by Lemma 4, is strict if there is a non-complete component of rank $r_{C}>4$.

If $\delta=0$, (3) implies $s_{F} \leq\left(\begin{array}{l}r \\ 2\end{array}\right)$ (equality only for $r=3$ ) as stated in Theorem 2. Indeed 
if $k \leq 2: \sum_{C \neq C^{\prime}} r_{C} r_{C^{\prime}}-k \geq r_{0}-k \geq r_{0}-2 \geq 0$, equality holding only for $F$ of type

$[2,1]$ (and $r=3$ );

if $k \geq 3: \sum_{C \neq C^{\prime}} r_{C} r_{C^{\prime}}-k \geq r_{0}(k-1)-k \geq 2(k-1)-k \geq 1$.

From now on we suppose $\delta=1$ : all non-complete $C \in \mathcal{C}$ are of linear type with odd ranks. In particular, we have $r_{0} \geq 3$. We write $G_{F}=C_{0} \cup C_{1} \cup \cdots \cup C_{m} \quad(m \geq 1)$ with $r_{0} \geq r_{1} \geq r_{m} \geq 1$ and $\sum\left|C_{i}\right|=n+1$.

We shall use for $r+1=r_{0}+r_{1}+\cdots+r_{m}$ and $\sum r_{C} r_{C^{\prime}}=r_{0} r_{1}+\cdots$ the estimations

$$
\begin{aligned}
r+1 & \geq r_{0}+k-1, \\
\sum r_{C} r_{C}^{\prime} & \geq r_{0}\left(r+1-r_{0}\right) .
\end{aligned}
$$

Note that the equality in (4) (resp. (5)) holds if and only if $F$ is of linear type $[3,1, \ldots, 1]$ (resp. $m=1$ ). We then obtain the estimation

$$
\left(\begin{array}{l}
r \\
2
\end{array}\right)-s_{F} \geq M
$$

with

$$
M=\left(r-r_{0}\right)\left(r_{0}-2\right)-2,
$$

where $r-r_{0}=r_{1}+\cdots+r_{m}-1 \geq m-1 \geq 0$ and $r_{0}-2 \geq 1$. We then have $M \geq-2$. We even obtain $M>0$ (i.e. $\left.s_{F}<\left(\begin{array}{c}r \\ 2\end{array}\right)\right)$ if $r-r_{0} \geq 3$. We now concentrate on the three cases $0 \leq r-r_{0} \leq 2$.

(a) $r=r_{0}: G_{F}=C_{0} \cup C_{1}$, with $r_{1}=1$. If $C_{0}$ is complete, $F$ is a canonical hyperplane and $s_{F}=\left(\begin{array}{c}r \\ 2\end{array}\right)+1$ as asserted in Theorem 2 . If $C_{0}$ is linear of rank $3,5,7, \ldots$, it follows from Lemma 3 that $s_{F}=1+s_{C_{0}}$ is equal to $1+\left(r_{0}+1\right)^{2} / 4=5,10,17, \ldots$, strictly smaller than $\left(\begin{array}{c}r \\ 2\end{array}\right)$ except for the cases $[3,1]$ (which realizes the maximum $s_{5}(3)$ ) and $[5,1]$ (which realizes $s_{n}(5)$ ), see figure 6 .

(b) $r=r_{0}+1: G_{F}=C_{0} \cup C_{1} \cup C_{2}$, with $r_{1}=r_{2}=1$. Since (5) is no more an equality, we obtain $\left(\begin{array}{c}r \\ 2\end{array}\right)-s_{F} \geq M+1=r_{0}-3 \geq 0$, where the equality requires that equality (4) holds, i.e. that $F$ is of type [3, 1, 1], which indeed realizes the maximum $s_{7}(4)=6$ (figure 7 ).

(c) $r=r_{0}+2$, i.e. $r_{1}+\cdots+r_{m}=3$. There are two occurrences of this situation: $m=3, r_{1}=r_{2}=r_{3}=1$, or $m=1, r_{1}=3$. In the first case, equality (5) does not

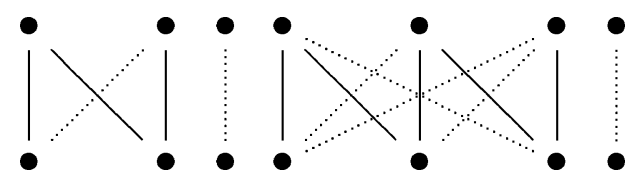

Figure 6. Linear types $[3,1]$ and $[5,1]$. 


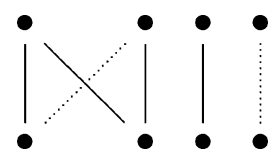

Figure 7. Type $[3,1,1]$.

hold. In the second case, (4) does not hold. Anyway, we have $\left(\begin{array}{l}r \\ 2\end{array}\right)-s_{F} \geq M+1=$ $2 r_{0}-5>0$.

This completes the proof of Theorem 2 .

\section{References}

1 C. Batut and J. Martinet, "Radiographie des réseaux parfaits", Experimental Math. 3 (1994), 39-49.

2 B. Bollobás, Modern Graph Theory, Graduate texts in Mathematics 184, Springer-Verlag, Heidelberg, 1998.

3 A.M. Bergé and J. Martinet, "Symmetric groups and lattices", Monatschefte für Math. 140(3) (2003), $179-195$.

4 J.H. Conway and N.J.A. Sloane, "Low-dimensional lattices. III. Perfect forms", Proc. Royal Soc. London A 418 (1988), 43-80.

5 J. Martinet, personal communication.

6 J. Martinet, Perfect Lattices in Euclidean Spaces, Grundlehren 327, Springer-Verlag, Heidelberg, 2003.

7 J. Martinet and B. Venkov, "On integral lattices having an odd minimum," Algebra and Analysis, SaintPetersburg 16(3) (2004), 198-237. 